(C) 2010 IEEE. Reprinted, with permission, from Li Li, Coprime factor model reduction for discrete-time uncertain systems . Decision and Control (CDC), 2010 49th IEEE Conference on , December 2010. This material is posted here with permission of the IEEE. Such permission of the IEEE does not in any way imply IEEE endorsement of any of the University of Technology, Sydney's products or services. Internal or personal use of this material is permitted.

However, permission to reprint/republish this material for advertising or promotional purposes or for creating new collective works for resale or redistribution must be obtained from the IEEE by writing to pubs-permissions@ieee.org. By choosing to view this document, you agree to all provisions of the copyright laws protecting it 


\title{
Coprime Factor Model Reduction for Discrete-time Uncertain Systems
}

\author{
$\mathrm{Li} \mathrm{Li}$
}

\begin{abstract}
This paper presents a contractive coprime factor model reduction approach for discrete-time uncertain systems of LFT form with norm bounded structured uncertainty. A systematic approach is proposed for coprime factorization and contractive coprime factorization of the underlying uncertain systems. The proposed coprime factor approach overcomes the robust stability restriction on the underlying systems which is required in the balanced truncation approach. The method is based on the use of LMIs to construct the desired reduced dimension uncertain system model.
\end{abstract}

\section{INTRODUCTION}

This paper addresses the coprime factorization (CF) and model reduction problems for discrete-time uncertain systems which are possibly robustly unstable. The uncertain systems under consideration are described in terms of linear fractional transformations (LFTs) with structured norm bounded uncertainty.

Model reduction has been an active research area in the control society since 1960s. One of the most commonly applied methods for stable linear time invariant (LTI) systems is the balanced truncation method [1] with guaranteed error bounds [2], [3]. For unstable LTI systems, a coprime factor approach [4] is proposed to avoid the stability issues. Discrete-time related topics can be found, for example, in [5] and the references therein.

Model reduction problems for uncertain systems have attracted much attention in recent years; see, for example, LFT systems [6], [7], [8], [9], [10], gain scheduling [11], linear parameter-varying systems [12], linear time-varying systems [13], nonlinear systems [14], linear parameter dependent (LPD) systems [15], and related approximation, truncation and simplification problems [16]. The balanced truncation method for robustly stable uncertain systems is studied in [6], [7] within the LFT framework. Concerning those uncertain systems which may be robustly unstable, a coprime factorization based approach is proposed in [9], which extends coprime factor approach [4] for LTI systems to the underlying uncertain systems. However, no indication is given in [9] on the contractiveness of the resulting coprime factors. This motivates the question as to whether a contractive $\mathrm{CF}$ can be obtained for uncertain systems. Contractive $\mathrm{CF}$, as an alternative to normalized $\mathrm{CF}$, has properties similar to normalized CF. In the meanwhile, it enables us to take advantage of linear matrix inequality (LMI) techniques, providing more flexibility to accommodate structure constraints including topological structures and uncertainty structures,

L. Li is with National ICT Australia, Department of Electrical and Electronic Engineering, The University of Melbourne, VIC, 3010, Australia li.lidee.unimelb.edu.au. and thus can be effectively solved by available softwares. Particularly for discrete-time uncertain systems, contractive $\mathrm{CF}$ is motivated by the following two observations. Firstly, for discrete-time LTI systems, applying balanced truncation to normalized coprime factors of original systems would result in contractive coprime factors of reduced systems, rather than normalized ones as in continuous cases. Therefore, it is not necessary to consider normalized $\mathrm{CF}$ in the first place in balanced truncation approaches. Secondly, in the presence of uncertainty, it is very difficult to obtain normalized coprime factors for the underlying systems because the corresponding Riccati equations are hard to solve and most probably leads to infeasible solutions.

In this paper, the coprime factor model reduction problem studied in [9] is revisited. The contribution of this paper, compared to the results of [9], are two folds. Firstly, the full column rank restriction on the B-matrix in [9] is eliminated , providing a more general solution to constructing coprime factorization for uncertain systems. Secondly, a systematic approach to obtain the coprime factorization for the underlying uncertain systems is presented based on the use of LMIs. A sufficient and necessary condition to ensure the feasibility of the derived LMI is also specified. Contractiveness is subsequently accomplished by choosing a specific feedback gain, which extends the similar LTI results to the uncertain systems under consideration. This enables us to apply balanced truncation [6] to the resulting contractive coprime factors to obtain the reduced-order uncertain systems. It is shown that the resulting reduced coprime factors are contractive as well. Although in this paper only uncertain systems are discussed, the results can be readily applied to multidimensional systems by replacing the uncertainty variables with frequency parameters.

Notation The notation is quite standard. $\mathbf{R}^{m \times n}$ and $\mathbf{C}^{m \times n}$ denote the set of real and complex, $m \times n$ matrices, and $\mathbf{H}^{m}$ denotes the set of Hermitian $m \times m$ matrices. Let $l^{m}$ and $l_{2}^{m}$ be the space of all the sequences and square summable sequences in $\mathbf{R}^{m}$ respectively. Let $\mathcal{L}\left(l^{m}\right)$ denote the space of all linear operators mapping from $l^{m}$ to $l^{m}$, and $L\left(l_{2}^{m}\right)$ denote the space of all linear bounded operators mapping from $l_{2}^{m}$ to $l_{2}^{m}$. The gain of an operator $\Delta$ in $\mathcal{L}\left(l_{2}^{m}\right)$ is given by $\|\Delta\|=$ $\sup _{z \in l_{2}^{m}, z \neq 0} \frac{\|\Delta z\|}{\|z\|}$, and the adjoint operator of $\Delta$ is denoted as $\Delta^{*}$ if $\Delta$ is linear, and if $\Delta=\Delta^{*}, \Delta<0$ means that $x^{*} \Delta x<0$ for any $x \neq 0$ in $\mathbf{R}^{m} . M^{*}$ is also used to denote the complex conjugate transpose of a complex matrix $M . F M(\cdot)^{*}$ and $(\cdot)^{*} M F$ denote $F M F^{*}$ and $F^{*} M F$ respectively for a Hermitian matrix $M$. 


\section{Problem Formulation}

We consider the uncertainty structure

$\boldsymbol{\Delta}^{\boldsymbol{c}}=\left\{\operatorname{diag}\left(\delta_{1} I_{h_{1}}, \cdots, \delta_{k} I_{h_{k}}\right): \delta_{i} \in \mathcal{L}\left(l_{2}\right), \delta_{i}\right.$ causal, $\left.\left\|\delta_{i}\right\| \leq 1\right\}$, and the following uncertain system:

$$
\mathcal{G}_{\Delta}:\left\{\begin{array}{l}
{\left[\begin{array}{l}
z \\
y
\end{array}\right]=\left[\begin{array}{ll}
A & B \\
C & D
\end{array}\right]\left[\begin{array}{l}
\xi \\
u
\end{array}\right],} \\
\xi=\Delta z, \quad \Delta \in \Delta^{c},
\end{array}\right.
$$

where $u(t) \in \mathbf{R}^{m}$ is the control input, $z(t) \in \mathbf{R}^{h}$ is the uncertainty output, $y(t) \in \mathbf{R}^{l}$ is the measured output and $\xi(t) \in \mathbf{R}^{h}$ is the uncertainty input; here $h=h_{1}+\cdots+h_{k}$. Similar to the typical setting for one-dimensional discretetime uncertain systems, we define $\delta_{1}=z^{-1}$, the time shift operator, and other $\delta_{i}$ 's are regarded as uncertainties.

Let the nominal system be denoted by $G=\left[\begin{array}{ll}A & B \\ C & D\end{array}\right]$. Then, the uncertain system (1) is defined by an LFT representation as follows. For any bounded linear operator $\Delta \in \mathcal{L}\left(l_{2}^{h}\right)$ such that $I-A \Delta$ is non-singular, define

$$
\mathcal{F}_{u}(G, \Delta):=D+C \Delta(I-A \Delta)^{-1} B .
$$

In what follows, robust stability, stabilizability and detectability of the uncertain system (1) are defined.

Definition 1 (Robust Stability [9]): The uncertain system (1) is said to be robustly stable, or equivalently, $\left(A, \Delta^{\boldsymbol{c}}\right)$ is said to be robustly stable, if $(I-A \Delta)^{-1}$ exists in $\mathcal{L}\left(l_{2}^{h}\right)$ and is causal, for all $\Delta \in \Delta^{c}$.

Definition 2: The uncertain system (1) is said to be robustly stabilizable if there exists a matrix $F$, such that $\left(A+B F, \Delta^{c}\right)$ is robustly stable. Similarly, the system (1) is said to be robustly detectable if the dual of the system (1) is robustly stabilizable.

The following lemma from [9] states a necessary and sufficient condition for robust stability. This lemma is given in terms of the positive commutant set corresponding to $\Delta^{c}$ defined as

$$
\boldsymbol{P}_{\boldsymbol{\Theta}}=\left\{\operatorname{diag}\left(\Theta_{1}, \cdots, \Theta_{k}\right): \Theta_{i} \in \mathbf{H}^{h_{i}}, \Theta_{i}>0\right\} .
$$

Lemma 3: (see [9, Proposition 3 and Remark 4]) The system (1) is robustly stable if and only if there exist $P \in \boldsymbol{P}_{\boldsymbol{\Theta}}$, such that

$$
A P A^{*}-P<0 .
$$

\section{BALANCED TRUNCATION}

In this section we briefly review the balanced truncation model reduction technique for the uncertain system (1) presented in [6]. It is assumed in this section that the uncertain system (1) is robustly stable. As in the LTI balanced truncation approach [1], [2], [3], this assumption is essential for the balanced truncation of the uncertain system (1), and guarantees the existence of the solutions to certain Lyapunov inequalities,

$$
\begin{aligned}
& A S A^{*}-S+B B^{*}<0, \\
& A^{*} P A-P+C^{*} C<0 .
\end{aligned}
$$

Theorem 4: The following statements are equivalent:

(i) The uncertain system (1) is robustly stable.

(ii) The LMI (4) admits a solution $S \in \boldsymbol{P}_{\boldsymbol{\Theta}}$.

(iii) The LMI (5) admits a solution $P \in \boldsymbol{P}_{\boldsymbol{\Theta}}$.

Definition 5: An uncertain system of the form (1) is said to be balanced if it has solutions to (4) and (5) which are identical diagonal matrices.

We summarize the proposed model reduction algorithm as follows.

\section{Procedure 6 (Balanced Truncation):}

1) Solve the LMIs (4) and (5) to obtain $S=$ $\operatorname{diag}\left(S_{1}, \cdots, S_{k}\right) \in \boldsymbol{P}_{\boldsymbol{\Theta}}, P=\operatorname{diag}\left(P_{1}, \cdots, P_{k}\right) \in \boldsymbol{P}_{\boldsymbol{\Theta}}$.

2) Balance $S_{i}, P_{i}$ by constructing a state transformation matrix $T_{i}[3]$ such that

$$
\begin{aligned}
& T_{i} S_{i} T_{i}^{*}=\left(T_{i}^{-1}\right)^{*} P_{i} T_{i}^{-1}=\Sigma_{i}=\operatorname{diag}\left(\Sigma_{i, 1}, \Sigma_{i, 2}\right) \\
& =\operatorname{diag}\left(\gamma_{1} I_{h_{i, 1}}, \ldots, \gamma_{d} I_{h_{i, d}}, \gamma_{d+1} I_{h_{i, d+1}}, \ldots, \gamma_{q} I_{h_{i, q}}\right) .
\end{aligned}
$$

Here $\gamma_{1}>\ldots>\gamma_{d}>\gamma_{d+1}>\ldots>\gamma_{q}>0$ are eigenvalues of $(X Y)^{1 / 2}$ with multiplicities $v_{j}, j=1, \ldots, q$ respectively; $h_{i, j} \geq 0$ satisfies $v_{j}=\sum_{i=1}^{k} h_{i, j}, j=1, \ldots, q$ and $h_{i}=\sum_{j=1}^{q} h_{i, j}, i=1, \ldots, k ; \Sigma_{i, 1}=\operatorname{diag}\left(\gamma_{1} I_{h_{i, 1}}, \ldots, \gamma_{d} I_{h_{i, d}}\right)$, $\Sigma_{i, 2}=\operatorname{diag}\left(\gamma_{d+1} I_{h_{i, d+1}}, \ldots, \gamma_{q} I_{h_{i, q}}\right)$.

3) Write the transformed nominal system of (1) as

$$
\bar{G}=\left[\begin{array}{cc}
\bar{A} & \bar{B} \\
\bar{C} & D
\end{array}\right],
$$

where

$$
\begin{aligned}
& \bar{A}=T A T^{-1}, \quad \bar{B}=T B, \\
& \bar{C}=C T^{-1}, \quad T=\operatorname{diag}\left(T_{1}, \cdots, T_{k}\right) .
\end{aligned}
$$

The sub-matrices of $\Delta$ and $\bar{G}$ corresponding to the matrix $\Sigma_{i, 2}, i=1, \ldots, k$ in (6) are truncated to obtain

$$
\begin{aligned}
G_{r} & =\left[\begin{array}{cc}
\bar{A}_{r} & \bar{B}_{r} \\
\bar{C}_{r} & D
\end{array}\right], \\
\Delta_{r} & =\operatorname{diag}\left(\delta_{1} I_{\tilde{h}_{1}}, \cdots, \delta_{k} I_{\tilde{h}_{k}}\right),
\end{aligned}
$$

where $\tilde{h}_{i}=\sum_{j=1}^{d} h_{i, j}, i=1, \ldots, k$.

4) Write the reduced dimension uncertain system as $\mathcal{G}_{r \Delta}=$ $\mathcal{F}_{u}\left(G_{r}, \Delta_{r}\right)$.

Theorem 7: Consider the uncertain system (1) and suppose that the reduced dimension uncertain system $\mathcal{G}_{r \Delta}$ is obtained as described in Procedure 6. Then $\mathcal{G}_{r \Delta}$ is also balanced and robustly stable. Furthermore,

$$
\sup _{\Delta \in \Delta^{c}}\left\|\mathcal{G}_{\Delta}-\mathcal{G}_{r \Delta}\right\| \leq 2\left(\gamma_{d+1}+\cdots+\gamma_{q}\right) .
$$

\section{Contractive Coprime Factor Model REDUCTION FOR UNCERTAIN SYSTEMS}

As introduced in the last section, the balanced truncation technique requires the underlying uncertain systems be robustly stable. For those uncertain systems which may be robustly unstable, one of the common approaches is coprime factorization approach. Coprime factorization of uncertain systems is explored in [9] for discrete-time systems, [10] for continuous-time system and [17] for parameter-dependent systems, and a model reduction algorithm based on coprime 
factorization is given in [9]. However, no indication is given in [9], [17] on the contractiveness of the obtained coprime factors. The balanced LQG truncation for uncertain systems is presented in [18]. It is shown in [19], [20] that the balanced LQG approach and coprime factor model reduction approach lead to identical reduced models in the continuoustime LTI cases. Inspired by these facts, in this section we follow the ideas in [18] to construct a contractive coprime factorization for uncertain systems of the form (1) and derive the corresponding model reduction algorithm.

\section{A. Coprime Factorization of Uncertain Systems}

Suppose that the uncertain system (1) is robustly stabilizable and robustly detectable, as stated in Def. 2. Consider the following LQG control and filter Riccati inequalities for the uncertain system (1),

$$
\begin{aligned}
& A^{*} W A-W+C^{*} C \\
& -\left(A^{*} W B+C^{*} D\right)\left(I+D^{*} D+B^{*} W B\right)^{-1}(\cdot)^{*}<0, \\
& A V A^{*}-V+B B^{*} \\
& -\left(A V C^{*}+B D^{*}\right)\left(I+D D^{*}+C V C^{*}\right)^{-1}(\cdot)^{*}<0
\end{aligned}
$$

in the variables $W \in \boldsymbol{P}_{\boldsymbol{\Theta}}, V \in \boldsymbol{P}_{\boldsymbol{\Theta}}$.

Note that the above Riccati inequalities have the same form as those LQG control and filter Riccati equations in the discrete-time LTI cases [21]; see also [22] for continuoustime cases. In these references, it is shown that the solutions to the LQG control and filter Riccati equations can be used for coprime factorization of the underlying LTI systems. In what follows, we will show that, similar to the LTI cases, the solutions of (12) and (13) can also be used to construct the coprime factors and contractive coprime factors of our uncertain systems (1).

Definition 8: Given a pair of uncertain systems $\mathcal{M}_{\Delta}=$ $\mathcal{F}_{u}(M, \Delta), \mathcal{N}_{\Delta}=\mathcal{F}_{u}(N, \Delta), \Delta \in \Delta^{\boldsymbol{c}}$, where $M$ and $N$ are constant matrices, $\left(\mathcal{M}_{\Delta}, \mathcal{N}_{\Delta}\right)$ is said to be a right coprime factorization (RCF) of $\mathcal{G}_{\Delta}(1)$ if the following conditions hold.

1) $\mathcal{M}_{\Delta}$ and $\mathcal{N}_{\Delta}$ are robustly stable.

2) For any fixed $\Delta \in \Delta^{c}, \mathcal{M}_{\Delta}$ is invertible in $\mathcal{L}\left(l^{m}\right)$ and $\mathcal{M}_{\Delta}^{-1}$ is casual.

3) For any fixed $\Delta \in \Delta^{c},\left(\mathcal{M}_{\Delta}, \mathcal{N}_{\Delta}\right)$ is right coprime, and $\mathcal{G}_{\Delta}=\mathcal{N}_{\Delta} \mathcal{M}_{\Delta}^{-1}$.

Furthermore, if $\mathcal{M}_{\Delta}^{*} \mathcal{M}_{\Delta}+\mathcal{N}_{\Delta}^{*} \mathcal{N}_{\Delta} \leq I$ for all $\Delta \in \Delta^{c}$, we say $\left(\mathcal{M}_{\Delta}, \mathcal{N}_{\Delta}\right)$ is a contractive RCF of $\mathcal{G}_{\Delta}(1)$.

Theorem 9: An uncertain system $\mathcal{G}_{\Delta}(1)$ is robustly stabilizable if and only if there exist matrices $\bar{P} \in \boldsymbol{P}_{\boldsymbol{\Theta}}$ and $X \in \mathbf{R}^{m \times h}$ solving the following LMI:

$$
\left[\begin{array}{cccc}
-\bar{P} & \bar{P} A^{*}+X^{*} B^{*} & X^{*} & \bar{P} C^{*}+X^{*} D^{*} \\
\star & -\bar{P} & \mathbf{0}_{h \times m} & \mathbf{0}_{h \times l} \\
\star & \star & -I_{m} & \mathbf{0}_{m \times l} \\
\star & \star & \star & -I_{l}
\end{array}\right]<0 .
$$

Furthermore, if $(\bar{P}, X)$ is a feasible solution to (14), then $\bar{P}^{-1}$ verifies (12).
Proof: (Only if part) Assume that the uncertain system $\mathcal{G}_{\Delta}$ in (1) is robustly stabilizable. From Definition 2 and Lemma 3 , there exist matrices $F$ and $P_{1} \in \boldsymbol{P}_{\boldsymbol{\Theta}}$, such that

$$
(A+B F)^{*} P_{1}(A+B F)-P_{1}<0 .
$$

Then we can apply Theorem 4 to show that there exists $P \in \boldsymbol{P}_{\boldsymbol{\Theta}}$ such that

$$
(A+B F)^{*} P(A+B F)-P+\left[\begin{array}{c}
C+D F \\
F
\end{array}\right]^{*}\left[\begin{array}{c}
C+D F \\
F
\end{array}\right]<0 .
$$

Define $\bar{P}=P^{-1}, X=F \bar{P}$. Left and right multiply both sides of (15) with $\bar{P}$, and we obtain

$(A \bar{P}+B X)^{*} \bar{P}^{-1}(A \bar{P}+B X)-\bar{P}+\left[\begin{array}{c}C \bar{P}+D X \\ X\end{array}\right]^{*}\left[\begin{array}{c}C \bar{P}+D X \\ X\end{array}\right]<0$.

It is easy to derive (14) by Schur complement from the above inequality.

(If part) Suppose that $(\bar{P}, X)$ is a feasible solution to (14). Defining $P=\bar{P}^{-1}, F=X P$, it is easy to show that (14) is equivalent to $(15)$. Therefore $\mathcal{G}_{\Delta}$ is robustly stabilizable.

Now we prove that $\bar{P}^{-1}$ verifies (12). By Schur complement, (14) is equivalent to

$-\bar{P}+X^{*} X+\left(\bar{P} C^{*}+X^{*} D^{*}\right)(\cdot)^{*}+\left(\bar{P} A^{*}+X^{*} B^{*}\right) \bar{P}^{-1}(\cdot)^{*}<0$,

which is

$$
\begin{aligned}
-\bar{P} & +\bar{P} C C^{*} \bar{P}+\bar{P} A^{*} \bar{P}^{-1} A \bar{P}-\left(\bar{P} C^{*} D+\bar{P} A^{*} \bar{P}^{-1} B\right) R^{-1}(\cdot)^{*} \\
& +\left[X^{*}+\left(\bar{P} C^{*} D+\bar{P} A^{*} \bar{P}^{-1} B\right) R^{-1}\right] R(\cdot)^{*}<0
\end{aligned}
$$

where $R=I+D^{*} D+B^{*} \bar{P}^{-1} B$.

Therefore, we have

$\bar{P} A^{*} \bar{P}^{-1} A \bar{P}-\bar{P}+\bar{P} C C^{*} \bar{P}-\left(\bar{P} C^{*} D+\bar{P} A^{*} \bar{P}^{-1} B\right) R^{-1}(\cdot)^{*}<0$.

It is clear that $\bar{P}^{-1}$ verifies (12).

Remark 10: The LMI (14) arises from the fact [23], [17] that the solution of the LQG control inequality (12) is related to a special state feedback $\mathcal{H}_{2}$ problem, that is, finding a static state feedback gain $F$, such that $\left\|\mathcal{F}_{l}\left(G_{S F \Delta}, F\right)\right\|_{\mathcal{H}_{2}}<\gamma$ with a given $\gamma>0$, where

$$
\mathcal{G}_{S F \Delta}=\mathcal{F}_{u}\left(G_{S F}, \Delta\right), \quad G_{S F}=\left[\begin{array}{ccc}
A & I & B \\
0 & 0 & I \\
C & 0 & D
\end{array}\right]
$$

here $\mathcal{F}_{l}(\cdot, \cdot)$ denotes the lower LFT representation. The LMI (14) is actually obtained by substituting $A, C$ in (5) with $A+$ $B F,\left[\begin{array}{c}C+D F \\ F\end{array}\right]$ respectively, letting $\bar{P}=P^{-1}, X=F \bar{P}$ and then applying Schur complement.

Theorem 11: Given an uncertain system $\mathcal{G}_{\Delta}(1)$ which is robustly stabilizable and robustly detectable, suppose matrices $\bar{P} \in \boldsymbol{P}_{\boldsymbol{\Theta}}$ and $X \in \mathbf{R}^{m \times h}$ satisfy the LMI (14). Let

$$
F=X \bar{P}^{-1}
$$


and consider the following system

$$
\begin{gathered}
\mathcal{G}_{F \Delta}=\left[\begin{array}{c}
\mathcal{N}_{\Delta} \\
\mathcal{M}_{\Delta}
\end{array}\right]=\mathcal{F}_{u}\left(G_{F}, \Delta\right), \\
G_{F}=\left[\begin{array}{cc}
A+B F & B \\
C+D F & D \\
F & I_{m}
\end{array}\right] .
\end{gathered}
$$

Then $\left(\mathcal{M}_{\Delta}, \mathcal{N}_{\Delta}\right)$ is an RCF of the uncertain system $\mathcal{G}_{\Delta}$.

Proof: From the proof of Theorem 9, it is clear that (14) is equivalent to (15). Thus $F$ is robustly stabilizing. Then invoke [9, Theorem 9] to complete the proof.

Remark 12: The coprime factorization presented in [9] requires that the matrix $B$ be of full column rank, such that a robustly stabilizing feedback gain can be obtained. The approach introduced in the above theorem removes such a restriction, and provides a systematic way to construct the coprime factors of the underlying uncertain systems. It is shown in the next section that, by picking up a specific statefeedback gain, this approach can be extended naturally to the contractive coprime factorization for our uncertain systems.

\section{B. Contractive Coprime Factorization of Uncertain Systems}

In the LTI case (without uncertainty), when seeking model reduction methods in the coprime factor description, we are particularly interested in normalized coprime factorizations, since robustness results on closed-loop stability are available. However, it is difficult, if not impossible, to find normalized coprime factorizations for the uncertain systems under consideration. Thus, in this section, contractive coprime factorizations are considered for uncertain systems of the form (1). We remark here that similar contractiveness ideas have been explored in [8], [24], [22], [10].

Theorem 13: Given a robustly stabilizable and detectable uncertain system $\mathcal{G}_{\Delta}(1)$, suppose there exist matrices $\bar{P} \in \boldsymbol{P}_{\boldsymbol{\Theta}}$ and $X \in \mathbf{R}^{m \times h}$ solving the LMI (14). Let

$$
\begin{aligned}
R & =I+D D^{*}+B^{*} \bar{P}^{-1} B, \\
F^{c} & =-R^{-1}\left(B^{*} \bar{P}^{-1} A+D^{*} C\right),
\end{aligned}
$$

and consider the following system,

$$
\begin{aligned}
\mathcal{G}_{F \Delta}^{c} & =\left[\begin{array}{c}
\mathcal{N}_{\Delta}^{c} \\
\mathcal{M}_{\Delta}^{c}
\end{array}\right]=\mathcal{F}_{u}\left(G_{F}^{c}, \Delta\right), \\
G_{F}^{c} & =\left[\begin{array}{cc}
A+B F^{c} & B R^{-\frac{1}{2}} \\
C+D F^{c} & D R^{-\frac{1}{2}} \\
F^{c} & R^{-\frac{1}{2}}
\end{array}\right] .
\end{aligned}
$$

Then $\left(\mathcal{M}_{\Delta}^{c}, \mathcal{N}_{\Delta}^{c}\right)$ is a contractive RCF of the uncertain system $\mathcal{G}_{\Delta}$.

Proof: Suppose the LMI (14) has a feasible solution $(\bar{P}, X)$. From the proof of Theorem 9, (14) is equivalent to (16). It is obvious that $\bar{P}, X^{c}=F^{c} \bar{P}$ also satisfy (16), therefore satisfies the LMI (14). It follows from Theorem 11 that $\left(\mathcal{M}_{\Delta}^{c}, \mathcal{N}_{\Delta}^{c}\right)$ is an RCF of $\mathcal{G}_{\Delta}$. Note that here $\left(\mathcal{M}_{\Delta}^{c}, \mathcal{N}_{\Delta}^{c}\right)$ are scaled by $R^{-\frac{1}{2}}$.

To prove that $\left(\mathcal{M}_{\Delta}^{c}, \mathcal{N}_{\Delta}^{c}\right)$ is contractive, that is $\left\|\mathcal{G}_{F \Delta}^{c}\right\| \leq 1$, we show an equivalent claim that $\left\|\mathcal{G}_{F \Delta}^{c}\right\|<\beta$ for any $\beta>1$.
By [25, Theorem 11.1], this claim is equivalent to find $P \in \boldsymbol{P}_{\boldsymbol{\Theta}}$ such that

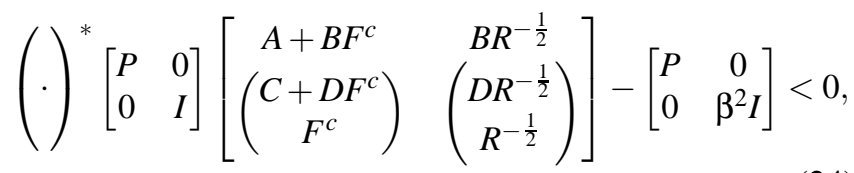

which is

$$
\left[\begin{array}{cc}
\mathcal{N}_{11} & \mathcal{N}_{12} \\
\star & \mathcal{N}_{22}
\end{array}\right]<0
$$

where

$$
\begin{aligned}
\mathcal{N}_{11}= & \left(A+B F^{c}\right)^{*} P\left(A+B F^{c}\right)-P+\left(F^{c}\right)^{*} F^{c} \\
& +\left(C+D F^{c}\right)^{*}\left(C+D F^{c}\right), \\
\mathcal{N}_{12}= & {\left[\left(A+B F^{c}\right)^{*} P B+\left(C+D F^{c}\right)^{*} D+\left(F^{c}\right)^{*}\right] R^{-\frac{1}{2}}, } \\
\mathcal{N}_{22}= & R^{-\frac{1}{2}}\left(-\beta^{2} R+I+D D^{*}+B^{*} P B\right) R^{-\frac{1}{2}} .
\end{aligned}
$$

It is easy to verify that $P=\bar{P}^{-1}$ satisfies (25). Indeed, substituting $P=\bar{P}^{-1}$ into (25), we have $\mathcal{N}_{12}=0$ and $\mathcal{N}_{11}<0$ by the fact that $\bar{P}, X^{c}=F^{c} \bar{P}$ also satisfy the LMI (14) and the fact that (14) is equivalent to (15); see proof of Theorem 11. Also, $\mathcal{N}_{22}=-\left(\beta^{2}-1\right) I<0$. Then (25) holds, and this completes the proof.

Remark 14: The construction of contractive coprime factors for the underlying uncertain systems is discussed in [8]. A two-step algorithm and an iteration algorithm are suggested. However, the overall conditions are not jointly convex in the decision variables, therefore there is no computationally tractable method to ascertain its feasibility. It is shown that Theorem 13 provides a tractable approach to the design of contractive coprime factors based on the use of LMIs. In the absence of uncertainty, the above results also recover those in the discrete-time LTI cases [21].

\section{Contractive Coprime Factor Model Reduction}

With the contractive RCF (22) in place, the model reduction method in [9] can be applied to the resulting contractive RCF. The only problem left is to compute the controllability and observability Gramians of the contractive RCF (22), as stated in the following theorem.

Theorem 15: Given that all the conditions in Theorem 13 are satisfied, the following statements hold.

(i) $\bar{P}^{-1}$ is a generalized observability Gramian for the uncertain system $\mathcal{G}_{F \Delta}^{c}(22)$.

(ii) The LMI

$$
\left(A+B F^{c}\right) S\left(A+B F^{c}\right)^{*}-S+B R^{-1} B^{*}<0
$$

has a feasible solution $S \in \boldsymbol{P}_{\boldsymbol{\Theta}}$ which is a generalized controllability Gramian for the uncertain system $\mathcal{G}_{F \Delta}^{c}$ (22).

Proof: (i) From the proof of Theorem 13, $\mathcal{N}_{11}<0$ in (25). This verifies that $P=\bar{P}^{-1}$ is a generalized observability Gramian for the uncertain system $\mathcal{G}_{F \Delta}^{c}(22)$.

(ii) Since $\mathcal{G}_{F \Delta}^{c}$ is robustly stable, invoking Theorem 4 , it is straightforward that the LMI (26) is feasible, and $S$ is a generalized controllability Gramian for $\mathcal{G}_{F \Delta}^{c}$. 
The above theorem provides a numerical way to compute generalized Gramians $P=\bar{P}^{-1}$ and $S$ for the contractive RCF $\left(\mathcal{M}_{\Delta}^{c}, \mathcal{N}_{\Delta}^{c}\right)$ of the uncertain system $\mathcal{G}_{\Delta}$. We are ready to summarize the proposed coprime factor model reduction algorithm as follows.

\section{Procedure 16 (Coprime Factor Model Reduction):}

1) Solve the LMI (14) to obtain $\bar{P}$. Define $R$ as in (20), $F^{c}$ as in (21) and $P=\bar{P}^{-1}$;

2) Solve the LMI (26) to obtain $S$;

3) Apply Steps 2-4 in Procedure 6 to the uncertain system $\mathcal{G}_{\Delta}(1)$ to obtain the reduced dimension uncertain system as $\mathcal{G}_{r \Delta}=\mathcal{F}_{u}\left(G_{r}, \Delta_{r}\right)$.

Assumption 17: The reduced system $\mathcal{G}_{r \Delta}=\mathcal{F}_{u}\left(G_{r}, \Delta_{r}\right)$ obtained in Procedure 16 is well-posed.

The discussion on the conditions of the well-posedness of the reduced system is beyond the scope of this paper. Here we will only concentrate on the model reduction technique for the uncertain systems. The reader can refer to [9] for some resorts, for example strict casuality on $\delta_{1} \times \cdots \times \delta_{k}$, guaranteeing a well-posed reduced system.

Theorem 18: Suppose that all the conditions in Theorem 13 are satisfied, and that the reduced dimension uncertain system $\mathcal{G}_{r \Delta}=\mathcal{F}_{u}\left(G_{r}, \Delta_{r}\right)$, where $G_{r}$ is defined in (9), is obtained as described in Procedure 16. Under Assumption 17, consider the following system,

$$
\begin{aligned}
\mathcal{G}_{r F \Delta}^{c} & =\left[\begin{array}{c}
\mathcal{N}_{r \Delta}^{c} \\
\mathcal{M}_{r \Delta}^{c}
\end{array}\right]=\mathcal{F}_{u}\left(G_{r F}^{c}, \Delta\right), \\
G_{r F}^{c} & =\left[\begin{array}{cc}
\bar{A}_{r}+\bar{B}_{r} F_{r}^{c} & \bar{B}_{r} R^{-\frac{1}{2}} \\
\bar{C}_{r}+D F_{r}^{c} & D R^{-\frac{1}{2}} \\
F_{r}^{c} & R^{-\frac{1}{2}}
\end{array}\right],
\end{aligned}
$$

where $F_{r}^{c}=-R^{-1}\left(\bar{B}^{*} \Sigma \bar{A}+D^{*} \bar{C}\right) \mathcal{P}^{*}$, and $\mathcal{P}$ is the corresponding truncation matrix in Procedure 16 defined as $\mathscr{P}=$ $\operatorname{diag}\left(\mathcal{P}_{1}, \cdots, \mathcal{P}_{k}\right), \mathcal{P}_{i}=\left[\mathbf{I}_{\tilde{h}_{i}} \mathbf{0}\right]$. Then the following statements hold.

(i) $\left(\mathcal{M}_{r \Delta}^{c}, \mathcal{N}_{r \Delta}^{c}\right)$ is a contractive RCF of $\mathcal{G}_{r \Delta}$.

(ii)

$$
\sup _{\Delta \in \Delta^{c}}\left\|\mathcal{G}_{F \Delta}^{c}-\mathcal{G}_{r F \Delta}^{c}\right\| \leq 2\left(\gamma_{d+1}+\cdots+\gamma_{q}\right)
$$

Proof: It follows the proof of [9, Theorem 11,] that $\left(\mathcal{M}_{r \Delta}^{c}, \mathcal{N}_{r \Delta}^{c}\right)$ is an RCF of $\mathcal{G}_{r \Delta}$ and the error bound (29) holds. It only remains to prove that $\left(\mathcal{M}_{r \Delta}^{c}, \mathcal{N}_{r \Delta}^{c}\right)$ is contractive.

For simplicity, here we only consider the case of $k=1$. As described in Procedure 16, let $T$ be the transformation matrix to balance $P, S$ and $\mathcal{P}=\left[\begin{array}{ll}I & 0\end{array}\right]$ be the corresponding truncation matrix. Then

$$
\begin{aligned}
T S T^{*} & =\left(T^{-1}\right)^{*} P T^{-1}=\operatorname{diag}\left(\Sigma_{1}, \Sigma_{2}\right), \\
\bar{A} & =T A T^{-1}=\left[\begin{array}{ll}
\bar{A}_{r} & \bar{A}_{12} \\
\bar{A}_{21} & \bar{A}_{22}
\end{array}\right], \quad \bar{B}=T B=\left[\begin{array}{l}
\bar{B}_{r} \\
\bar{B}_{2}
\end{array}\right], \\
\bar{C} & =C T^{-1}=\left[\begin{array}{ll}
\bar{C}_{r} & \bar{C}_{2}
\end{array}\right] .
\end{aligned}
$$

Define the following matrices, partitioned accordingly with the partition in (30),

$$
\begin{gathered}
\mathcal{A}=\bar{A}+\bar{B} F^{c} T^{-1}=\left[\begin{array}{ll}
\mathcal{A}_{11} & \mathcal{A}_{12} \\
\mathcal{A}_{21} & \mathcal{A}_{22}
\end{array}\right], \mathcal{B}=\bar{B} R^{-\frac{1}{2}}=\left[\begin{array}{c}
\mathcal{B}_{1} \\
\mathcal{B}_{2}
\end{array}\right], \\
\mathcal{C}=\left[\begin{array}{c}
\bar{C}+D F^{c} T^{-1} \\
F^{c} T^{-1}
\end{array}\right]=\left[\begin{array}{ll}
\mathcal{C}_{1} & \mathcal{C}_{2}
\end{array}\right], \quad \mathcal{D}=\left[\begin{array}{c}
D R^{-\frac{1}{2}} \\
R^{-\frac{1}{2}}
\end{array}\right] .
\end{gathered}
$$

Note that, from the proof of Theorem 13, (24) holds for $P=\bar{P}^{-1}$. Left- and right-multiply both sides of (24) with $\operatorname{diag}\left(\left(T^{-1}\right)^{*}, I\right)$ and $\operatorname{diag}\left(T^{-1}, I\right)$, and we have

$$
(\cdot)^{*}\left[\begin{array}{cc}
\Sigma & 0 \\
0 & I
\end{array}\right]\left[\begin{array}{cc}
\mathcal{A} & \mathcal{B} \\
\mathcal{C} & \mathcal{D}
\end{array}\right]-\left[\begin{array}{cc}
\Sigma & 0 \\
0 & \beta^{2} I
\end{array}\right]<0 .
$$

Left- and right-multiply both sides of (31) with $\operatorname{diag}(\mathscr{P}, I)$ and $\operatorname{diag}\left(\mathscr{P}^{*}, I\right)$ to obtain

$$
\begin{aligned}
& (.)^{*}\left[\begin{array}{cc}
\Sigma_{1} & 0 \\
0 & I
\end{array}\right]\left[\begin{array}{cc}
\mathcal{A}_{11} & \mathcal{B}_{1} \\
\mathcal{C}_{1} & \mathcal{D}
\end{array}\right]-\left[\begin{array}{cc}
\Sigma_{1} & 0 \\
0 & \beta^{2} I
\end{array}\right] \\
& <-(\cdot)^{*} \Sigma_{2}\left[\begin{array}{ll}
\mathcal{A}_{21} & \mathcal{B}_{2}
\end{array}\right]<0 .
\end{aligned}
$$

It is easy to verify that $\mathcal{A}_{11}, \mathcal{B}_{1}, \mathcal{C}_{1}, \mathcal{D}$ are the system matrices of $G_{r F}^{c}$ in (28). Therefore $\left(\mathcal{M}_{r \Delta}^{c}, \mathcal{N}_{r \Delta}^{c}\right)$ is contractive; see the proof of Theorem 13.

Remark 19: Note that solutions to the LMIs (14) and (26), if they exist, are not unique. In the absence of uncertainty, minimizing $\bar{P}^{-1}$ subject to (14) and minimizing $S$ subject to (26) could lead to a normalized coprime factor model reduction algorithm. A possible heuristic is to seek approximate normalization in the presence of structured uncertainty, that is, solve the following two semi-definite programs:

$$
\begin{aligned}
& \min \operatorname{trace}(Z) \text { with } \bar{P} \in P_{\Theta} \\
& \text { subject to (14) and }\left[\begin{array}{cc}
Z & I \\
I & \bar{P}
\end{array}\right]>0, \\
& \text { min } \operatorname{trace}(S) \text { with } S \in P_{\Theta} \text { subject to (26). }
\end{aligned}
$$

Also note that although the two conditions in (14) and (26) are convex (separately), however, similar to the balanced truncation in the LTI case, the computation of a solution of the LMI constraints which leads to the best reduced model introduces a non-convex problem.

\section{EXAMPLE}

Consider the same example in [9] with

$$
\begin{aligned}
A & =\left[\begin{array}{ccccc}
0.5034 & 0.1768 & -0.2340 & -0.1406 & 0.5814 \\
0.0096 & 0.5498 & -0.0362 & -0.6744 & 2.2496 \\
0.0337 & 0.2546 & 0.0984 & -0.4051 & 1.3599 \\
-0.2709 & 0.1470 & 0.3249 & 0.0484 & 0.6356 \\
-0.0909 & 0.0491 & 0.1075 & -0.1019 & 0.5681
\end{array}\right], \\
B & =\left[\begin{array}{ll}
0.3306 & 0.1700 \\
0.8951 & 0.3442 \\
0.5487 & 0.2143 \\
0.8748 & 0.8821 \\
0.5217 & 0.4479
\end{array}\right], \\
C & =\left[\begin{array}{lllll}
3.0622 & -0.9986 & -0.7126 & 6.4339 & -10.4291 \\
3.0396 & -0.9913 & -0.7073 & 5.2369 & -8.4887
\end{array}\right], \\
\Delta & =\operatorname{diag}\left(\delta_{1} I_{3}, \delta_{2} I_{2}\right) .
\end{aligned}
$$


Solving the LMI (14), we obtain

$$
\begin{aligned}
P=\bar{P}^{-1} & \\
= & {\left[\begin{array}{ccccc}
96.1453 & -24.7774 & -30.4131 & 0.0000 & 0.0000 \\
-24.7774 & 7.5136 & 6.4620 & 0.0000 & 0.0000 \\
-30.4131 & 6.4620 & 12.5545 & 0.0000 & 0.0000 \\
0.0000 & 0.0000 & 0.0000 & 98.5330 & -163.4846 \\
0.0000 & 0.0000 & 0.0000 & -163.4846 & 275.9870
\end{array}\right], } \\
F^{c}= & {\left[\begin{array}{ccccc}
1.2027 & -0.1404 & -0.5680 & 0.8741 & -2.2285 \\
-0.0957 & -0.2160 & -0.2979 & -1.0129 & 1.5355
\end{array}\right] . }
\end{aligned}
$$

We can then construct the contractive RCF as in (22), (23) with

$M_{F}^{c}=\left[\begin{array}{ccccc|cc}0.8847 & 0.0937 & -0.4724 & -0.0238 & 0.1057 & 0.1758 & 0.0555 \\ 1.0532 & 0.3498 & -0.6471 & -0.2406 & 0.7834 & 0.4883 & 0.0886 \\ 0.6731 & 0.1313 & -0.2771 & -0.1425 & 0.4662 & 0.2990 & 0.0560 \\ 0.6968 & -0.1663 & -0.4347 & -0.0804 & 0.0406 & 0.4197 & 0.3764 \\ 0.4937 & -0.1209 & -0.3222 & -0.0996 & 0.0933 & 0.2585 & 0.1829 \\ \hline 3.0622 & -0.9986 & -0.7126 & 6.4339 & -10.4291 & 0.0000 & 0.0000 \\ 3.0396 & -0.9913 & -0.7073 & 5.2369 & -8.4887 & 0.0000 & 0.0000 \\ 1.2027 & -0.1404 & -0.5680 & 0.8741 & -2.2285 & 0.5860 & -0.1053 \\ -0.0957 & -0.2160 & -0.2979 & -1.0129 & 1.5355 & -0.1053 & 0.5311\end{array}\right]$

Solving the LMI (26), we obtain

$$
S=\left[\begin{array}{lllll}
0.0772 & 0.1973 & 0.1214 & 0.0000 & 0.0000 \\
0.1973 & 0.5222 & 0.3203 & 0.0000 & 0.0000 \\
0.1214 & 0.3203 & 0.1968 & 0.0000 & 0.0000 \\
0.0000 & 0.0000 & 0.0000 & 0.5599 & 0.3169 \\
0.0000 & 0.0000 & 0.0000 & 0.3169 & 0.1827
\end{array}\right]
$$

Then the balanced Gramian is

$\Sigma_{1}=\operatorname{diag}(0.8370,0.3146,0.0268), \Sigma_{2}=\operatorname{diag}(1.1436,0.8147)$.

Truncating the system matrices corresponding to the last generalized Hankel singular value in $\Sigma_{1}$, the reduced dimension uncertain system model is defined by

$$
\begin{aligned}
& \bar{A}_{r}=\left[\begin{array}{cccc}
0.3830 & 0.0865 & -0.3771 & 0.7548 \\
-0.1633 & 0.6255 & -0.0157 & 0.1556 \\
-0.6731 & 0.5150 & 0.3777 & -0.2194 \\
-0.3692 & 0.2832 & 0.0094 & 0.2388
\end{array}\right], \\
& \bar{B}_{r}=\left[\begin{array}{cc}
1.0572 & 0.0349 \\
-0.2642 & -0.5105 \\
-0.6643 & -1.5790 \\
0.9864 & 0.0412
\end{array}\right] \text {, } \\
& \bar{C}_{r}=\left[\begin{array}{llll}
-0.2745 & -0.0669 & -0.6423 & -0.2425 \\
-0.2725 & -0.0664 & -0.5228 & -0.1973
\end{array}\right] \text {, } \\
& \Delta_{r}=\operatorname{diag}\left(\delta_{1} I_{2}, \delta_{2} I_{2}\right) \text {. }
\end{aligned}
$$

and the error bound on the coprime factors is given by

$$
\sup _{\Delta \in \Delta^{c}}\left\|\mathcal{G}_{F \Delta}^{c}(s)-\mathcal{G}_{r F \Delta}^{c}(s)\right\| \quad \leq 2 \times 0.0268=0.0536 .
$$

\section{CONCLUSIONS}

The paper considers the problem of coprime factor model reduction for a class of discrete-time uncertain systems with structured norm bounded uncertainty. The proposed method is applicable to the uncertain systems which may be robustly unstable, overcoming the robust stability restriction in the balanced truncation approach. A systematic approach is presented to construct a contractive coprime factor for the underlying uncertain system, based on the used of LMIs. This enables the balanced truncation to be applied to the contractive coprime factor to obtain the reduced uncertain system.

\section{REFERENCES}

[1] B. Moore, "Principal component analysis in linear systems: controllability, observability, and model reduction," IEEE Transactions on Automatic Control, vol. 26, pp. 17-32, 1981.

[2] D. Enns, "Model reduction with balanced realizations: an error bound and frequency weighted generalization," in Proceedings of the $23 \mathrm{rd}$ IEEE Conference on Decision and Control, 1984, pp. 127-132.

[3] K. Glover, "All optimal Hankel-norm approximations of linear multivariable systems and their $L_{\infty}$ error bounds," International Journal of Control, vol. 39, pp. 1115-1193, 1984.

[4] D. Meyer, "Fractional balanced reduction: model reduction via fractional representation," IEEE Trans. Automat. Contr., vol. 35, no. 12, pp. 1341-1345, Dec. 1990.

[5] D. Hinrichsen and A. J. Pritchard, "An improved error estimate for reduced-order models of discrete-time systems," IEEE Trans. Automat. Contr., vol. 35, no. 3, pp. 317-320, 1990.

[6] C. Beck, J. Doyle, and K. Glover, "Model reduction of multidimensional and uncertain systems," IEEE Transactions on Automatic Control, vol. 41, no. 10, pp. 1466-1477, 1996.

[7] L. Li and I. R. Petersen, "A Gramian-based approach to model reduction for uncertain systems," in Proceedings of the 46th IEEE Conference on Decision and Control, 2007, pp. 4373-4378.

[8] C. Beck and P. Bendottii, "Model reduction methods for unstable uncertain systems," in Proceedings of the 36th IEEE Conference on Decision and Control, vol. 4, 1997, pp. 3298-3303.

[9] C. Beck, "Coprime factors reduction methods for linear parameter varying and uncertain systems," Systems \& Control Letters, vol. 55, no. 3, pp. 199-213, 2006.

[10] L. Li, "Coprime factor model reduction for continuous-time uncertain systems," in Proceedings of the 46th IEEE Conference on Decision and Control, Cancun, Mexico, 2008, pp. 4227-4232.

[11] P. Apkarian and P. Gahinet, "A convex characterization of gainscheduled $H_{\infty}$ controllers," IEEE Transactions on Automatic Control, vol. 40, no. 5, pp. 853-864, 1995 .

[12] G. Wood, P. Goddard, and K. Glover, "Approximation of linear parameter-varying systems," in Proceedings of the 35th IEEE Conference on Decision and Control, vol. 1, 1996, pp. 406-411.

[13] S. Lall and C. Beck, "Error-bounds for balanced model-reduction of linear time-varying systems," IEEE Transactions on Automatic Control, vol. 48, no. 6, pp. 946-956, 2003.

[14] Y.-C. Chu and K. Glover, "Bounds of the induced norm and model reduction errors for systems with repeated scalar nonlinearities," IEEE Transactions on Automatic Control, vol. 44, no. 3, pp. 471-483, 1999.

[15] L. Li, "Model reduction for linear parameter-dependent systems," in Proceedings of the 17th IFAC World Congress, Seoul, Korea, 2008, pp. 4048-4053.

[16] L. Andersson, A. Rantzer, and C. Beck, "Model comparison and simplification," International Journal of Robust and Nonlinear Control, vol. 9, no. 3, pp. 157-181, 1999.

[17] E. Prempain, "On coprime factors for parameter-dependent systems," in Proceedings of 45th IEEE Conference on Decision and Control, 2006, pp. 5796-5800.

[18] L. Li and I. R. Petersen, "A Gramian-based approach to model reduction for uncertain systems," IEEE Transactions on Automatic Control, vol. 55, no. 2, pp. 508-514, 2010.

[19] R. Ober and D. McFarlane, "Balanced canonical forms for minimal systems: a normalized coprime factor approach," Linear Algebra \& its Applications, vol. 122-124, pp. 23-64, 1989.

[20] L. Li, Structured model reduction and control for interconnected systems. Ph.D. Thesis, University of California, Los Angeles, 2005.

[21] V. Ionescu and C. Oara, "A discrete-time reduced-order controller for robust stabilization of plant in the normalized coprime-factor description," IMA J Math Control Info, vol. 11, no. 3, pp. 231-252, 1994.

[22] L. Li and F. Paganini, "Structured coprime factor model reduction based on LMIs," Automatica, vol. 41, no. 1, pp. 145-151, 2005.

[23] J. C. Doyle, K. Glover, P. P. Khargonekar, and B. A. Francis, "Statespace solutions to standard $H_{2}$ and $H_{\infty}$ control problems," IEEE Transactions on Automatic Control, vol. 34, no. 8, pp. 831-847, 1989.

[24] C. Beck and J. Doyle, "Model reduction of behavioural systems," in Proceedings of the 32nd IEEE Conference on Decision and Control, vol. 4, 1993, pp. 3652-3657.

[25] G. E. Dullerud and F. Paganini, A Course in Robust Control Theory : a Convex Approach. New York: Springer, 2000. 